\title{
Chemical synthesis of oligosaccharide mimetics
}

\author{
Pierre Sinay \\ Ecole Normale Supérieure, Département de Chimie, URA CNRS 1686 \\ 24, Rue Lhomond, 75231 Paris Cedex 05, France
}

\begin{abstract}
A variety of $C$-disaccharides have been selectively and expeditiously synthesized through the so-called tether approach.
\end{abstract}

\section{Introduction}

Oligosaccharides are involved in a increasing number of biological processes. This has stimulated the development of selective methods for the synthesis of such complex molecules. Among others, the imidate procedure that we have discovered (ref. 1), and which has been remarkably modified as the trichloroacetimidate version (ref. 2), is of classical use in the field of glycosylation. A host of synthetic complex oligosaccharides are thus easily available for the benefit of glycobiology. Knowledge of their geometrical and motional properties in aqueous solution is important for understanding the structureactivity relationship and for an appreciation of the ease of taking-up of the active conformation - the one matching the corresponding receptor and inducing an optimal biological response-. A potential way to subtly alter such a conformation is to replace the interglycosidic oxygen atom by a methylene group. In doing so, the exo anomeric effect is removed. A comparison of recent studies (ref. 3) has shown that the effect of such a replacement on geometrical and motional properties of di or oligosaccharide is a matter of discussion: It should also be noted that such disaccharide close mimetics are not degraded by glycosidases, a feature which could be of practical interest if one's try to develop an oligosaccharide based drug.

The purpose of this invited lecture is to report on recent advances on the development of a synthetic entry to $\mathrm{C}$-disaccharides, based on a 8 or 9 endo-trig radical cyclisation reaction from two monosaccharide temporarily connected through a tether. Silicon tethered reactions have gained increasing general importance for the successful synthesis of a variety of complex molecules (ref. 4). The practical use of the temporary silicon connector in the carbohydrate field has been materialized by Stork within the context of C-glycoside synthesis (ref. 5).

\section{Stereoselective synthesis of $\alpha$ and $\beta C$-disaccharides}

Galactose and galactosamine are monosaccharides which are frequently found in natural oligosaccharides of biological importance. As previously shown by us (ref. 6), we can modulate the $\alpha / \beta$ selectivity of the $C$-galactosylation through the position of tethering (Scheme 1). 

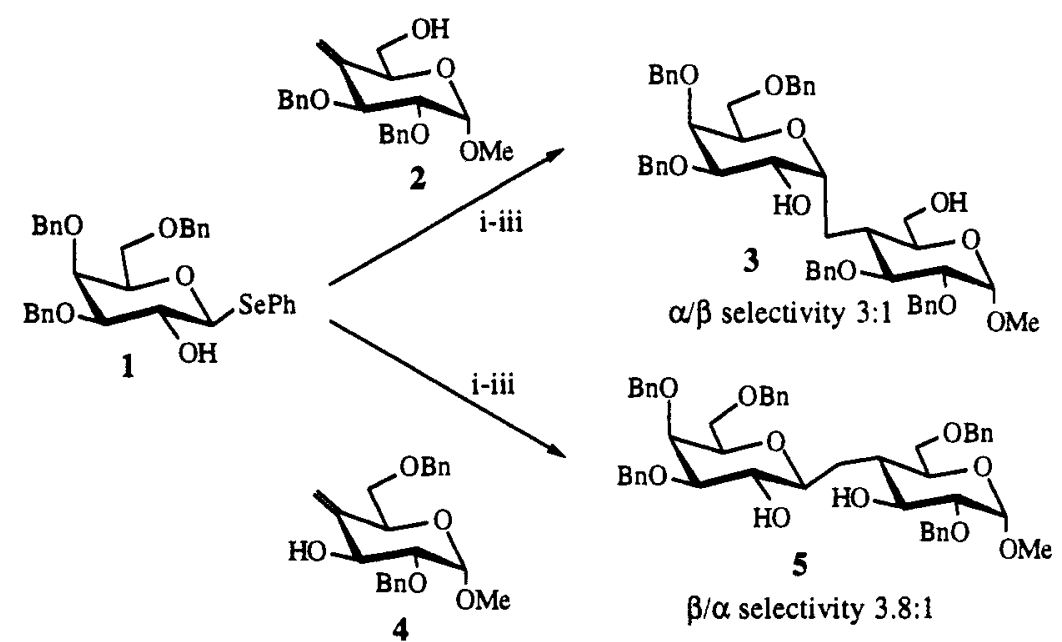

$\beta / \alpha$ selectivity $3.8: 1$

Scheme 1: Reagents: i) 1)1, BuLi, 2) $\mathrm{Me}_{2} \mathrm{SiCl}_{2}$; ii) 2 or 4, imidazole, THF; iii) Bu 3 SnH, toluene, reflux, then aq. HF/ THF.

When the galactopyranosyl donor 1 was tethered with the primary hydroxyl group of 2 , selective $\alpha-\mathrm{C}$ galactosylation was observed, via a 9 endo-trig cyclisation process, to give compound 3 . When the same donor 1 was now tethered with alcohol 4, the selectivity of the C-galactosylation of C-4 was nicely reversed, and the protected C-lactoside derivative $\mathbf{5}$ was now obtained. Although this work is basically interesting in conceptual terms, the selectivity was rather limited and we sought for a much more selective approach. Scheme 2 describes a highly efficient and stereoselective example of an $\alpha-C$ galactosylation. The galactosyl donor 1 was replaced by the donor 6 , which was tethered with alcohol 7 through a diisopropyl silicon tether.
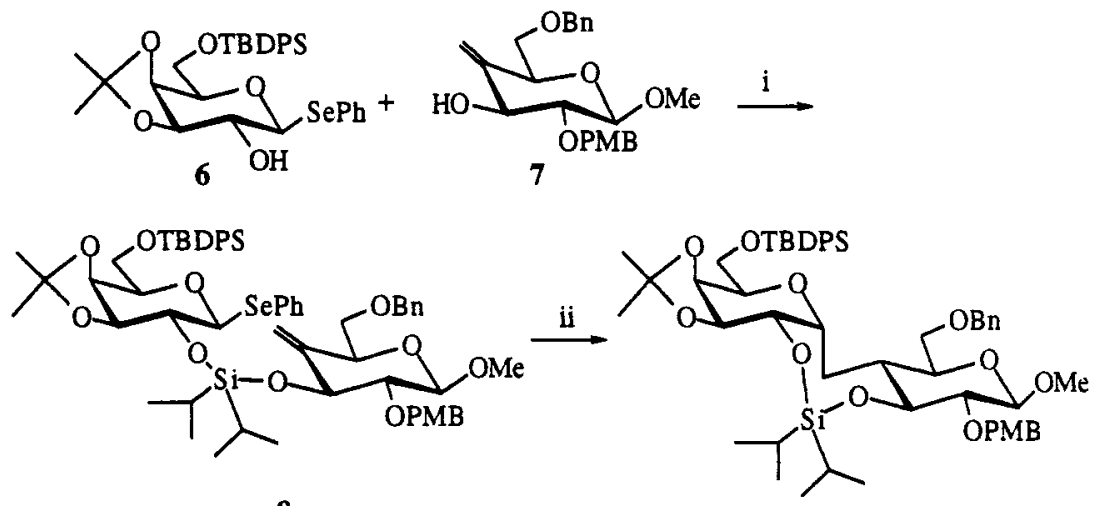

8

9

Scheme 2: Reagents: i) 1) 6, BuLi, THF, $\left.0^{\circ} \mathrm{C}, 2\right) \mathrm{iPr}_{2} \mathrm{SiCl}_{2}, 4$ equiv., $-78^{\circ} \mathrm{C}$ to r. t., 3) Concentrate to dryness, 4) 7, DMAP, THF, r. t.(70\%); ii) Bu3snH, AIBN, toluene, reflux (80\%).

This tether, which is more stable than the previous one (dimethyl), allows the easy isolation by flash chromatography of the silaketal 8 and thus a precise evaluation (80\%) of the yield of the radical cyclisation. A remarkable feature of this reaction is its remarkable double selectivity, the $\alpha$-Cdisaccharide 9 being the single product of the rection. This exquisite $\alpha$-C-galactosylation has been explained by B. Giese (personal communication), which has shown (scheme 3) that the model radical 10 adopts a boat conformation, so that only $\alpha$-C-galactosylation can occur for geometrical reasons. In 
contrast, a model 11 of the previously used C-galactosyl donor adopts a ${ }^{4} C_{1}$ chair conformation, resulting in an non-unexpected moderate selectivity.

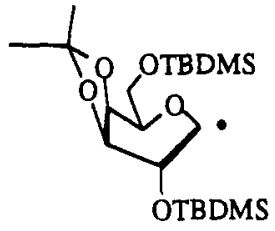

10

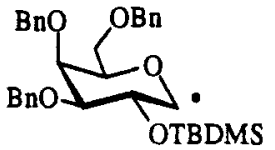

11

\section{Scheme 3}

The other selectivity as been explained as clearly shown in scheme 4 by a 1,5 hydrogen transfer from the primary benzyl group.

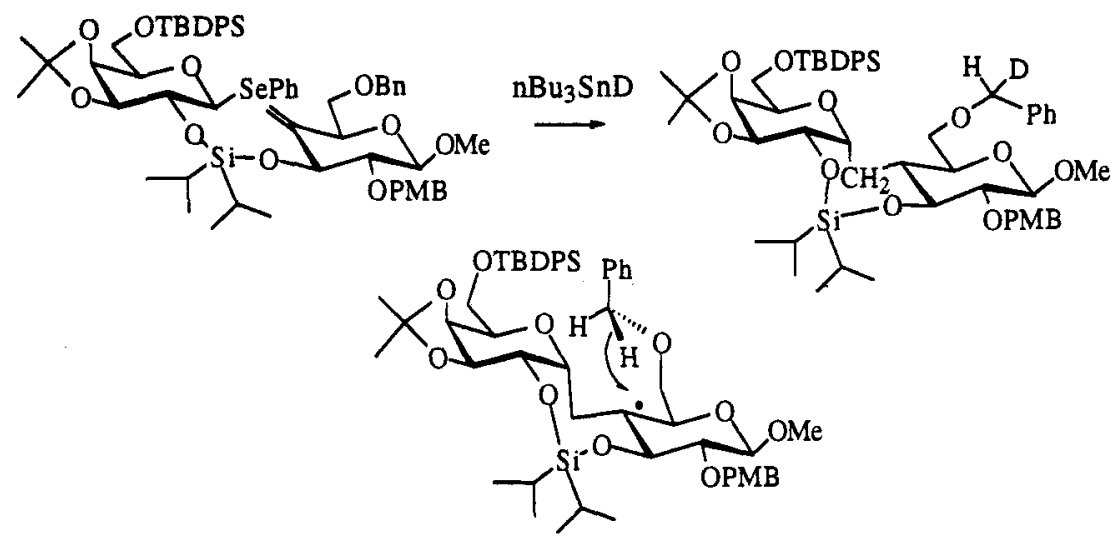

Scheme 4

In order to solve the problem of the direct $C$-galactosaminylation, we worked out a temporary phosphoramidic connection as typically shown in scheme 5 :
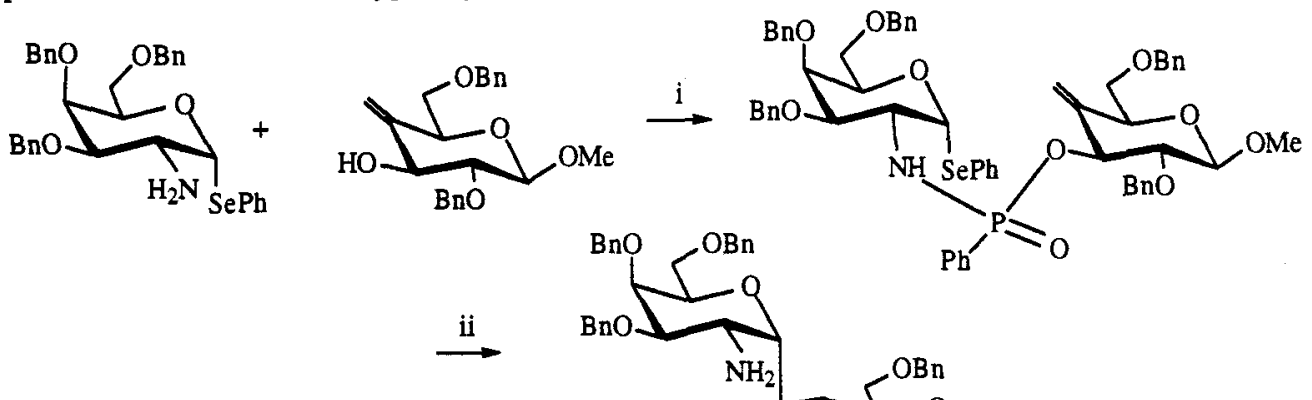

$\alpha / \beta 3 / 2$

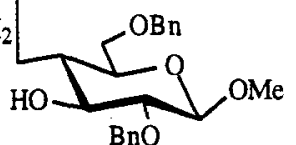

Scheme 5 : Reagents: i) 1) $\mathrm{PhPCl}_{2}, \mathrm{Et}_{3} \mathrm{~N}$, THF, r. t.(80\%); 2) $\mathrm{tBuOOH}$; ii) 1) $\mathrm{Bu}_{3} \mathrm{SnH}$, AIBN, toluene, reflux, 2) $\mathrm{LiAlH}_{4}$, THF (47\%).

Finally, we have also used another approach, whereby an anomeric exoglucal is connected, through a silaketal tether, to an iodosugar, as shown in scheme 6: 


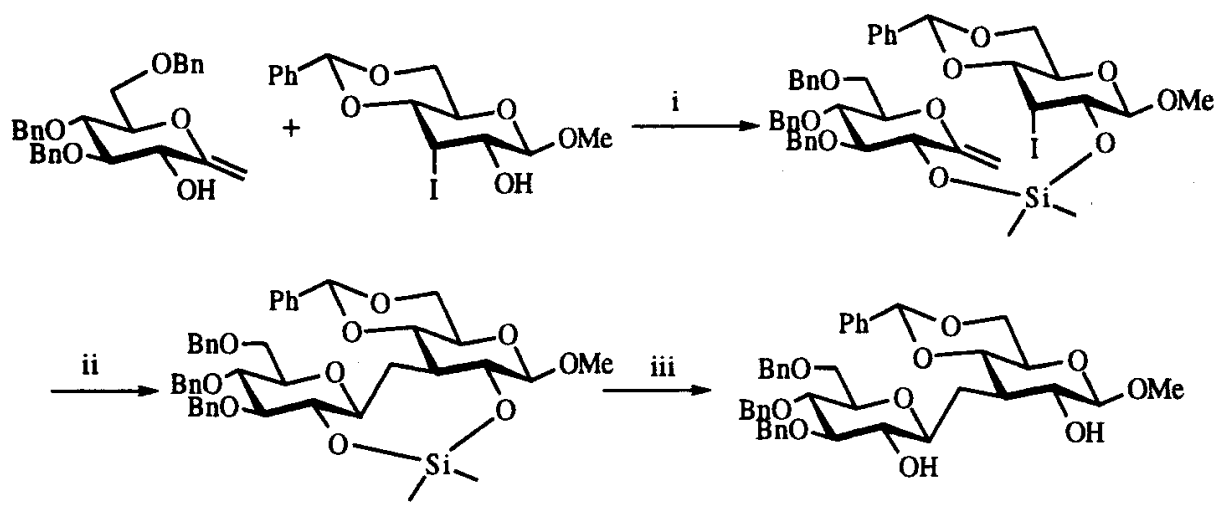

Scheme 6 : Reagents: i) BuLi, $\mathrm{Me}_{2} \mathrm{SiCl}_{2}$; ii) $\mathrm{Bu}_{3} \mathrm{SnH}, \mathrm{AIBN}$, toluene, reflux, then nBu4NF (40\%).

In this case, the resulting anomeric radical is expectedly reduced from the $\alpha$-side to selectively provide a $\beta$-C-disaccharide.

\section{Conclusion}

This lecture reports a selection of selective syntheses of $C$-disaccharides we have recently achieved in our group, clearly detronstrating the potentiality of the tethering approach for the practical synthesis of carbohydrate mimetics of biological relevance. A study of their biological properties is currently underway.

\section{Acknowledgements}

The lecturer would like to thank Dr. Jean-Maurice Mallet for the preparation of this manuscript and for his invaluable participation to this research programme. The selected examples have been studied by $\mathrm{E}$. Untersteller, B. La Ferla, G. Rubinstenn, J. Esnault, and B. Vauzeilles. Part of this work has been done in collaboration with Professor B. Giese (Basel).

\section{References}

1. J.-R. Pougny, J.-C. Jacquinet, M. Nassr, D. Duchet, M.-L. Milat, P. Sinaÿ, J. Am. Chem. Soc. 99, 6762 (1977).

2. R. R. Schmidt, J. Michel, Angew. Chem. Int. Ed. Engl. 19,731 (1980).

3. a) Y. Wang, P. G. Goekjian, D. M. Ryckmann, W. H. Miller, S. A. Babirad, Y. Kishi, J. Org. Chem. 57, 482 (1992); b) J.-F. Espinosa, M. Martín-Pastor, J. L. Asensio, H. Dietrich, M. Martín-Lomas, R. R. Schmidt, J. Jiménez-Barbero, Tetrahedron Lett. 36, 6329 (1995); c) A. Wei, A. Haudrechy, C. Audin, H. -S. Jun, N. Haudrechy-Bretel, Y. Kishi, J. Org. Chem. 60, 2160 (1995); d) P. Berthault, N. Birliradis, G. Rubinstenn, P. Sinaÿ, H. Desvaux, J. Biolmol. NMR 8, 23 (1996); e) J.-F. Espinosa, F. J. Cañada, J. L. Asencio, M. Martín-Pastor, H. Dietrich, M. Martín-Lomas, R. R. Schmidt, J. Jiménez-Barbero, J. Am. Chem. Soc. 118, 10862 (1996).

4. M.Bols, T. Skrydstrup, Chem Rev. 95, 1253 (1995).

5. G. Stork, H. S. Suh, G. Kim, J. Am. Chem. Soc. 113, 7054 (1991).

6. a) A. Mallet, J.-M. Mallet, P. Sinaÿ, Tetrahedron Asym. 2593 (1994); b) P. Sinaÿ, Pure \& Appl Chem. in press. 